\title{
EL SISTEMA DE ALBAÑILERÍA INTEGRAL ALLWALL CON $\mathrm{BHH} / \mathrm{BLOC}+$
}

\author{
(THE ALLWALL INTEGRAL MASONRY SYSTEM WITH BHH/BLOC+)
}

Josep M Adell

Profesor, Doctor Arquitecto. Universidad Politécnica Madrid (UPM). Dpto. Construcción y Tecnología Arquitectónicas (DCTA). Escuela Técnica Superior de Arquitectura (ETSAM). Director Línea Investigación sobre fábricas en el Grupo de Investigación de Tecnologías Innovadoras y Sostenibles en Edificación (TISE-fábricas). Presidente Delegación España SC-6.

Benito Lauret

Doctor Arquitecto. Profesor Titular (DCTA-UPM-ETSAM-TISE)

Fecha de recepción: 20-I-05

ESPAÑA

876-10

\section{RESUMEN}

Se desarrolla a continuación, una nueva técnica constructiva para levantar muros de fábrica armados en las 3 direcciones del espacio, cualquiera que sea el material empleado y utilizando sólo mortero en lugar de hormigón.

\section{El Sistema de Albañilería Integral AllWall, está} especialmente ideado para facilitar la ejecución por parte del albañil, y economizar mano de obra, al tiempo que controla la fisuración de la fábrica e incrementa las aplicaciones técnicas de las fábricas.

La Asociación de Fabricantes de Hormigón española, NORMABLOC, está potenciando el sistema constructivo AllWall, con su nuevo bloque de hormigón BLOC+.

\section{SUMMARY}

A new construction theory is discussed, based on the erection of masonry walls that can be reinforced in all three spatial dimensions, regardless of the material employed and using plain mortar instead of concrete.

The AllWall integral masonry system is especially designed to facilitate the mason's work and lower labour costs, while controlling wall cracking and broadening the scope of application of masonry technique.

NORMABLOC, the Spanish association of concrete manufacturers, is encouraging the use of the AllWall building system with its new concrete block, BLOC+.

\section{INTRODUCCIÓN}

El SAI: SISTEMA DE ALBAÑILERÍA INTEGRAL ${ }^{\circledR}$ es una nueva técnica constructiva de reciente desarrollo, que permite armar vertical y horizontalmente cualquier tipo de fábrica, con independencia del tipo de material empleado (macizo, hueco, perforado, ladrillo o bloque, cerámica u hormigón), logrando construir placas armadas en las 3 direcciones del espacio, con cualquiera de dichos materiales.

La técnica de "LA FÁBRICA ARMADA: Murfor ${ }^{\circledR}$ ", habitualmente empleada en España desde que fue desarrollada en 1992 por el que suscribe, parte de emplear cerchas de tendel homogéneamente distribuidas en un muro, con cerchas de tendel cada $60 \mathrm{~cm}$ de altura como máximo, y con una proporción del $0,03 \%$ de acero en la sección de la fábrica para controlar su fisuración, empleando mortero en lugar de hormigón (Fig. 1).

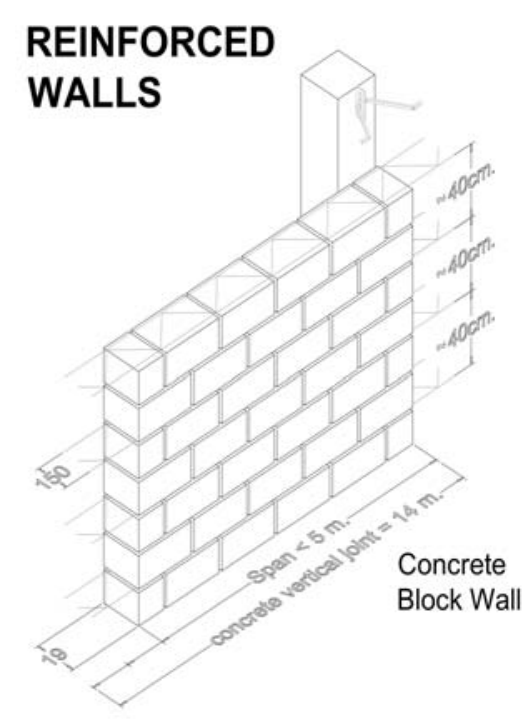

Figura 1.- Muro de Fábrica Armada con Bloque de Hormigón. 


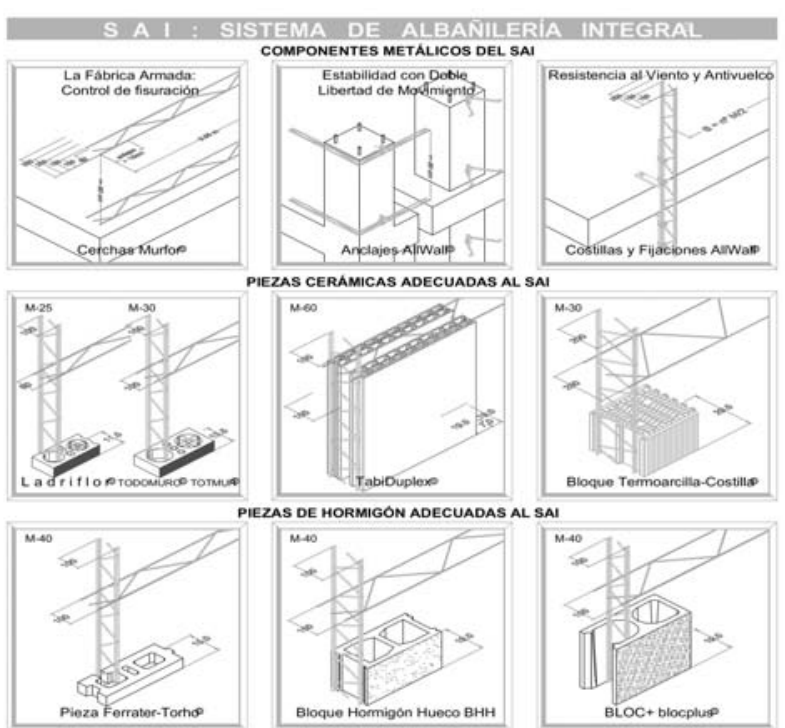

Figura 2.- SAI: El Sistema de Albañilería Integral (AllWall).

Este nuevo enfoque se presentó en Construmat'03 (Barcelona) y en el stand de Normabloc en la Feria Construtec'04 (Madrid) (Fig.3).

Recientemente se ha desarrollado un nuevo bloque denominado BLOC + que tiene la particularidad de facilitar la puesta en obra del armado vertical de los muros por acceso lateral, al armado previamente situado en vertical (Fig.4.a,b).

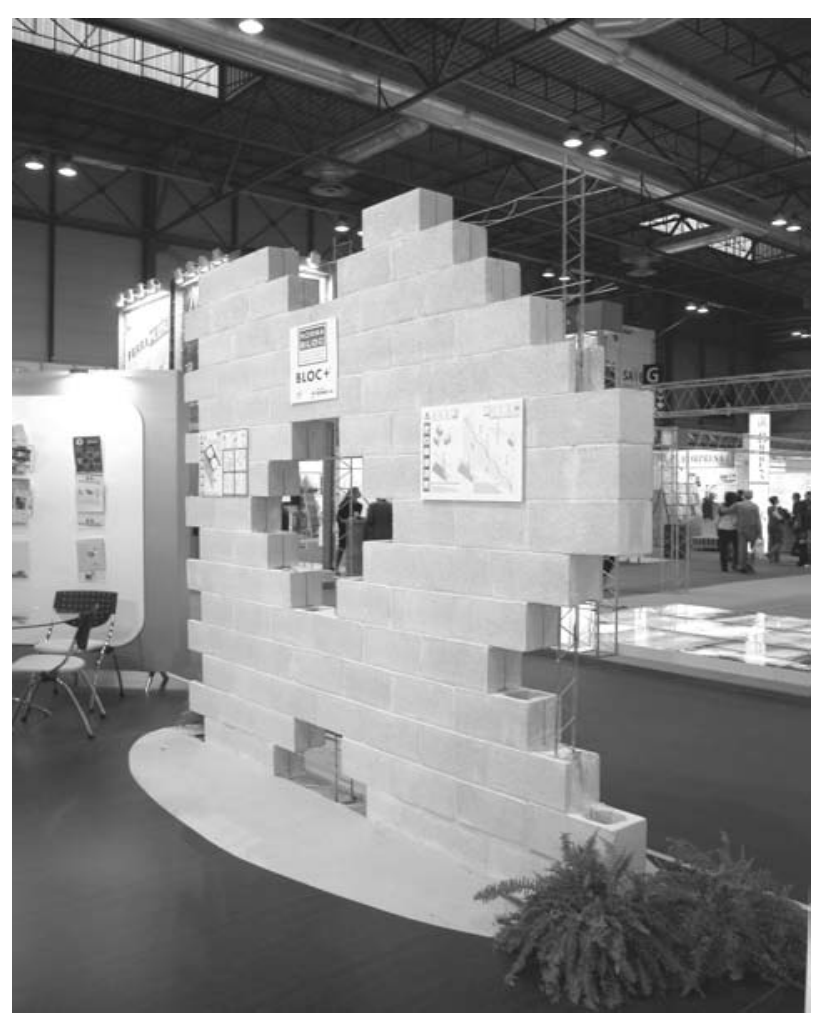

Figura 3.- Vista del stand Normabloc en la Feria Construtec, Madrid 2004.

\section{EL SAI ${ }^{\circledR}:$ SISTEMA DE ALBAÑILERÍA INTE- GRAL $^{\circledR}$ ALLWALL ${ }^{\circledR}$}

El SISTEMA DE ALBAÑILERÍA INTEGRAL ${ }^{\circledR}$ añade a "la fábrica armada" la posibilidad de armar los muros verticalmente con o sin mortero, ya que las costillas que se emplean son autorresistentes, y no requieren del mismo para transmitir sus esfuerzos entre sí (pues se sujetan arriba $\mathrm{y}$ abajo de los forjados con las correspondientes fijaciones del sistema).

El elemento más novedoso, con Patente de Invención de J.M. Adell (1997), es la Costilla ${ }^{\circledR}$ Vertical de Refuerzo AllWall ${ }^{\circledR}$, que consiste en una celosía doble capaz de enhebrarse horizontalmente con las armaduras de tendel en forma de cercha.

En la ficha SAI: Sistema de Albañilería Integral ${ }^{\circledR}$ : AllWall ${ }^{\circledR}$ (Fig.2), se expone la síntesis del Sistema.

Los componentes metálicos del SAI son:

- Cerchas de tendel tipo Murfor ${ }^{\circledR}$.

- Anclajes AllWall ${ }^{\circledR}$ de diversas libertades de movimiento.

- Costillas AllWall ${ }^{\circledR}$ con sus fijaciones específicas.

Las piezas cerámicas adecuadas al SAI son:

- Ladriflor $^{\circledR}$ métrico TodoMuro ${ }^{\circledR}$ y Ladriflor $^{\circledR}$ catalán TotMur ${ }^{\circledR}$

- Muro doble de piezas de gran formato Tabiduplex ${ }^{\circledR}$

- $\quad$ Bloque Termoarcilla para Costilla ${ }^{\circledR}$ )

Las piezas de hormigón adecuadas al SAI son:

- Pieza Ferrater-Torho ${ }^{\circledR}$

- Bloque de Hormigón Hueco BHH

- $\quad$ BlocPlus $^{\circledR}$, Bloc $^{\circledR}{ }^{\circledR}$

Según se arme un muro con mayor o menor proporción de armaduras de tendel y/o costillas, se logrará cumplimentar eficazmente las solicitaciones propias del tipo de muro que se construya.

Los componentes del $\mathrm{SAI}^{\circledR}$ se comercializan con la denominación AllWall ${ }^{\circledR}$ (TodoMuro ${ }^{\circledR}$ ), adaptándose, como ya se ha dicho, a cualquier material y tipología de muro, ya que para cada ancho de fábrica existen los anchos apropiados de la cercha horizontal y la costilla vertical.

\section{TÉCNICA DE EJECUCIÓN}

NORMABLOC, la nueva asociación nacional que agrupa a los fabricantes de bloques y mampostería de hormigón, quiere promover la calidad de sus materiales así como las nuevas técnicas constructivas que facilitan su colocación y empleo. 

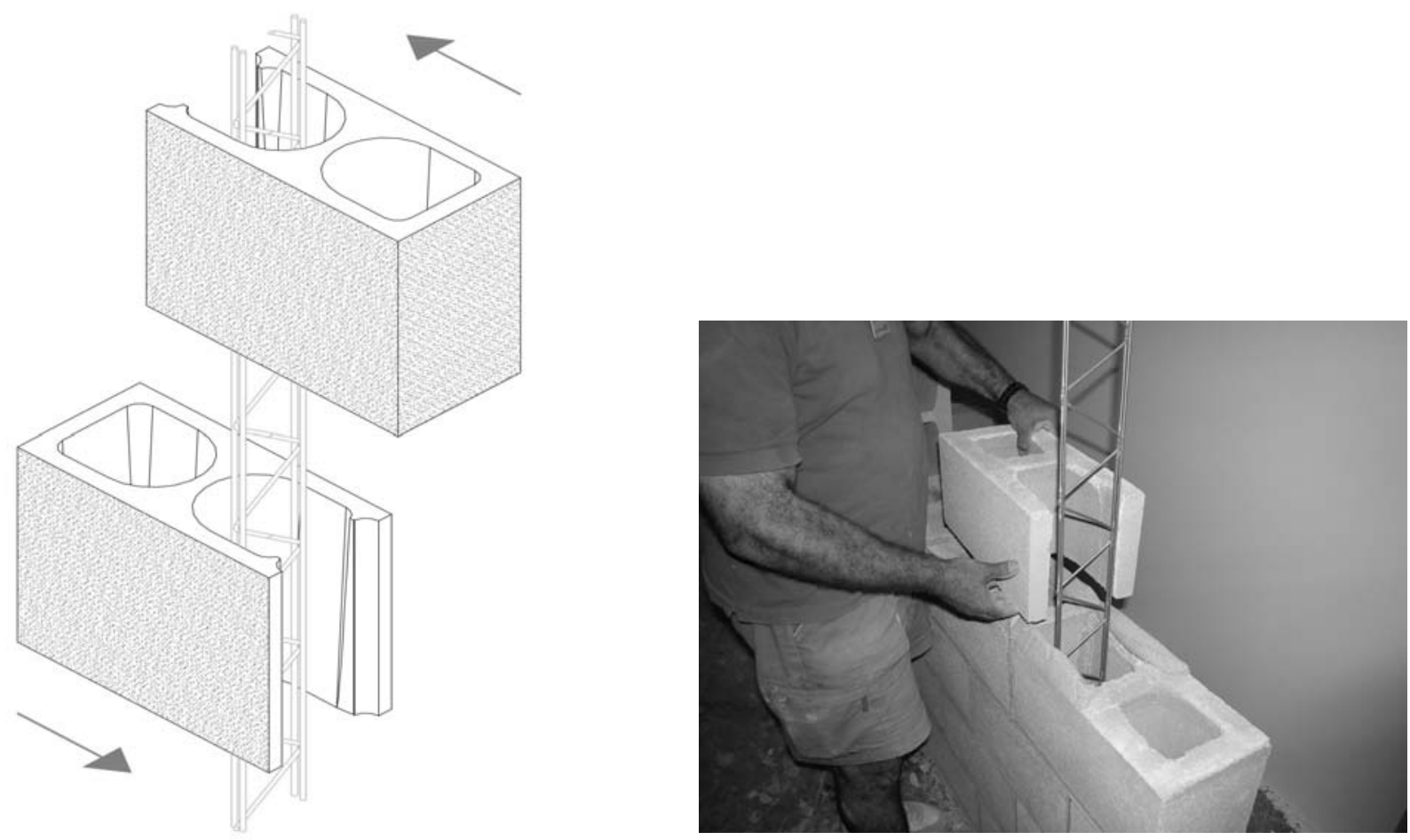

Figura 4.- a) Gráfico del procedimiento de acceso lateral del BLOC+ para albergar la costilla vertical AllWall en su interior; b) Foto del proceso de introducción del bloque dentro de la costilla.

El SAI AllWall/BLOC+, contempla junto con la pieza de Bloque de Hormigón Hueco BLOC+, desarrollada por Normabloc, las costillas verticales con sus fijaciones en los extremos, cerchas de tendel tipo Murfor, además de anclajes AllWall con una o dos libertades de movimiento para muros entestados o pasantes según como se sujeten al soporte.

Para lograr el entrecruzado de los armados vertical y horizontal, las costillas verticales, se enhebran con las armaduras de tendel, para lo que suele aprovecharse realiza el solape de estas últimas en la vertical de las primeras (Fig.5.a,b,c,d).

El sistema AllWall contempla, además, las fijaciones inferiores y/o superiores de las costillas para asegurar la transmisión de esfuerzos a los forjados (Fig.6.a,b,c).

Los extremos de los muros suelen acometer a los soportes de la estructura porticada existente, siendo común que los cerramientos sean pasantes por delante de la estructura, mientras que las particiones acometan directamente a los pilares. Para lograr la libertad de movimiento a la hora de anclar el muro a los soportes, el Sistema AllWall ofrece anclajes con doble libertad de movimiento para los cerramientos pasantes, y con una libertad de movimiento, para las particiones entestadas (Fig.7.a,b).

El Sistema AllWall/BLOC + ofrece dos posibilidades de ubicación de las costillas: dentro de las piezas o entre las piezas.
Con las costillas ubicadas dentro del $\mathrm{BLOC}+$, no se interrumpe el aparejo del muro y no se aprecia dónde está el armado vertical ubicado, empleándose para muros de fábrica vista de bloque de hormigón (Fig.8.a,b).

Con las costillas ubicadas en la llaga vertical continua, aunque está perfectamente trabada con las cerchas horizontales, la llaga queda aparente en la vertical del armado, teniendo la ventaja de poder emplear costillas más anchas y, por lo tanto, de mayor inercia frente al cálculo de resistencia al viento, lo que no afecta estéticamente en muros interiores o para revocar (Fig.9.a,b).

Construyendo con el SAI, se levantan muros que constituyen placas estructurales que tienen armado homogéneo con cerchas horizontales cada 40 ó $60 \mathrm{~cm}$ como máximo (2 ó 3 hiladas de bloques) y armado vertical de costillas distanciadas normalmente cada 7 bloques, equivalente a 2,80 m para muros de cerramiento (como los cortafuegos del Aeropuerto de Barajas) y cada 14 bloques, equivalente a $5,6 \mathrm{~m}$ para particiones interiores industriales (Fig.10.a,b).

Una vez decidida la ubicación de las costillas y replanteadas éstas en función de los bloques, se disponen en vertical fijadas inferiormente al forjado, para posteriormente, levantar la fábrica entre ellas, introduciendo las piezas dentro de los bloques, o dejando las costillas en llagas verticales continuas, aunque perfectamente trabadas horizontalmente con la fábrica armada (Fig.11.a,b). 

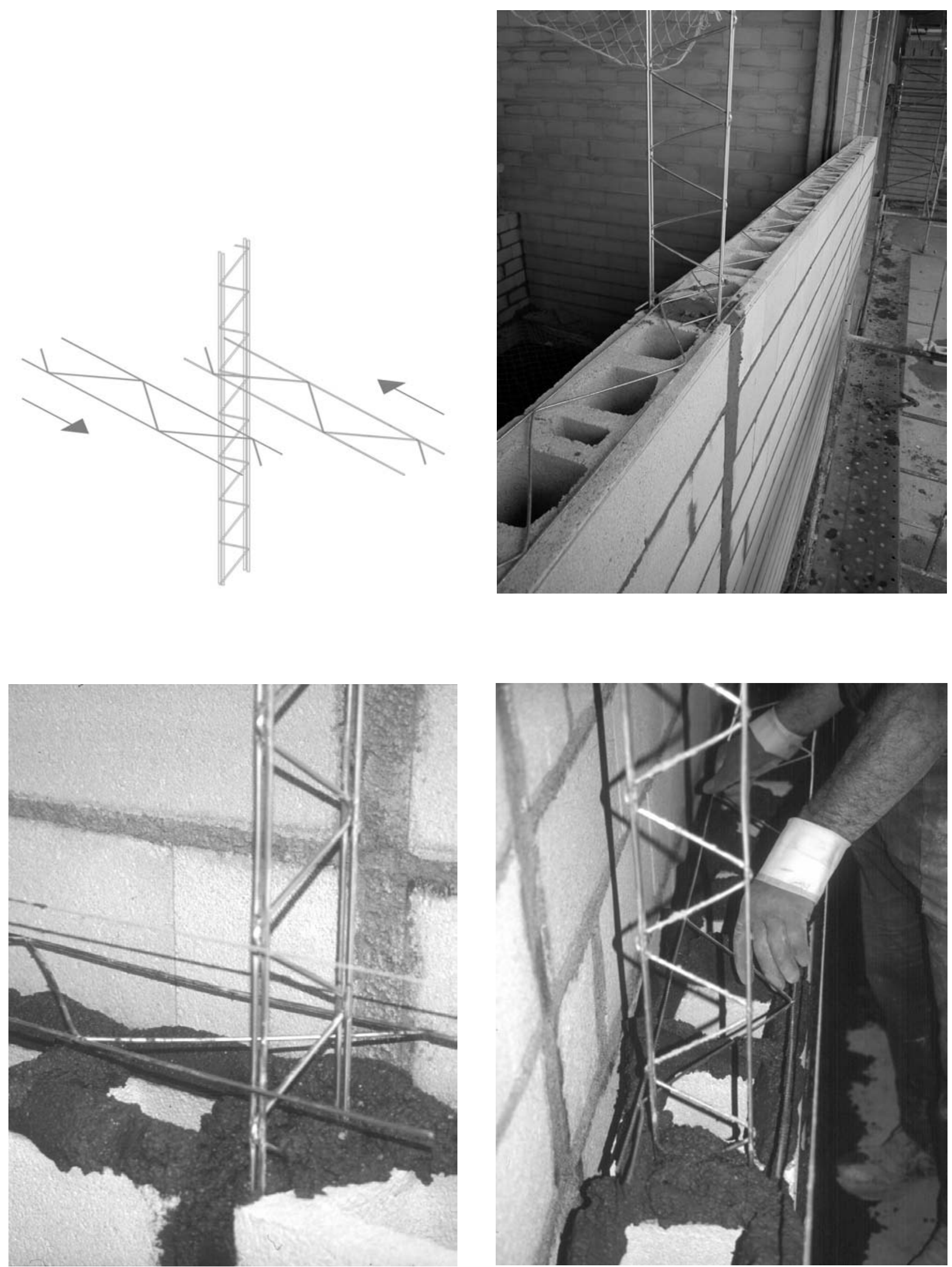

Figura 5.- a) Gráfico del armado tridimensional del SAI por combinación de las cerchas horizontales con las costillas verticales por solape; b) Replanteo del solape de las cerchas Murfor en la vertical de la costilla; c) Solapado del armado Murfor del paño izquierdo acometiendo a la Costilla AllWall; d) Solapado de la armadura Murfor del paño derecho acometiendo a la costilla AllWall. 


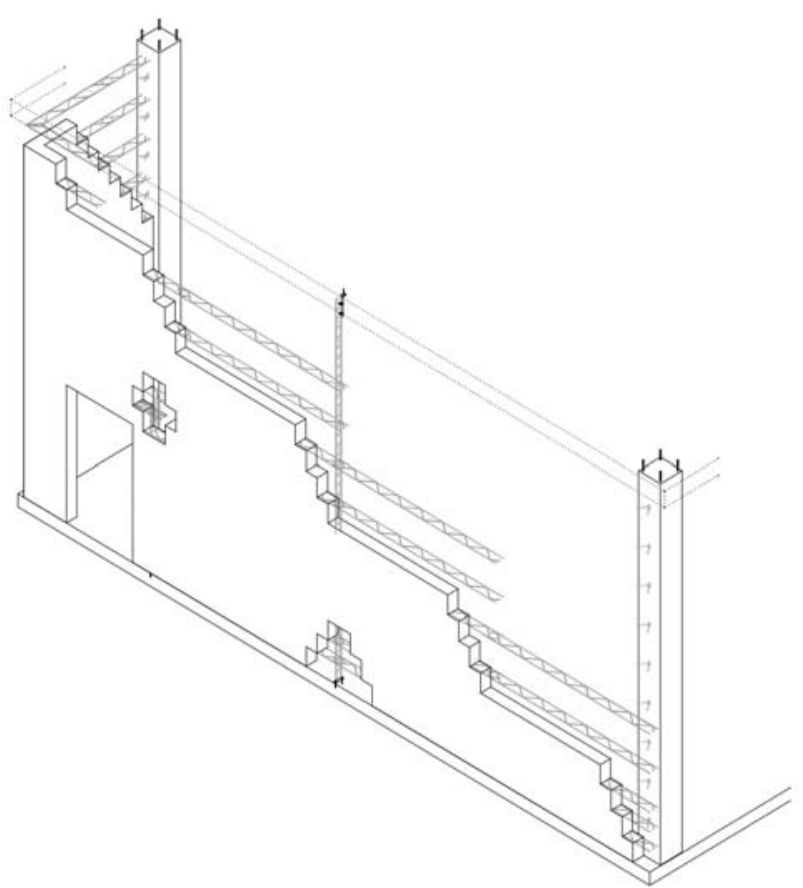

a)

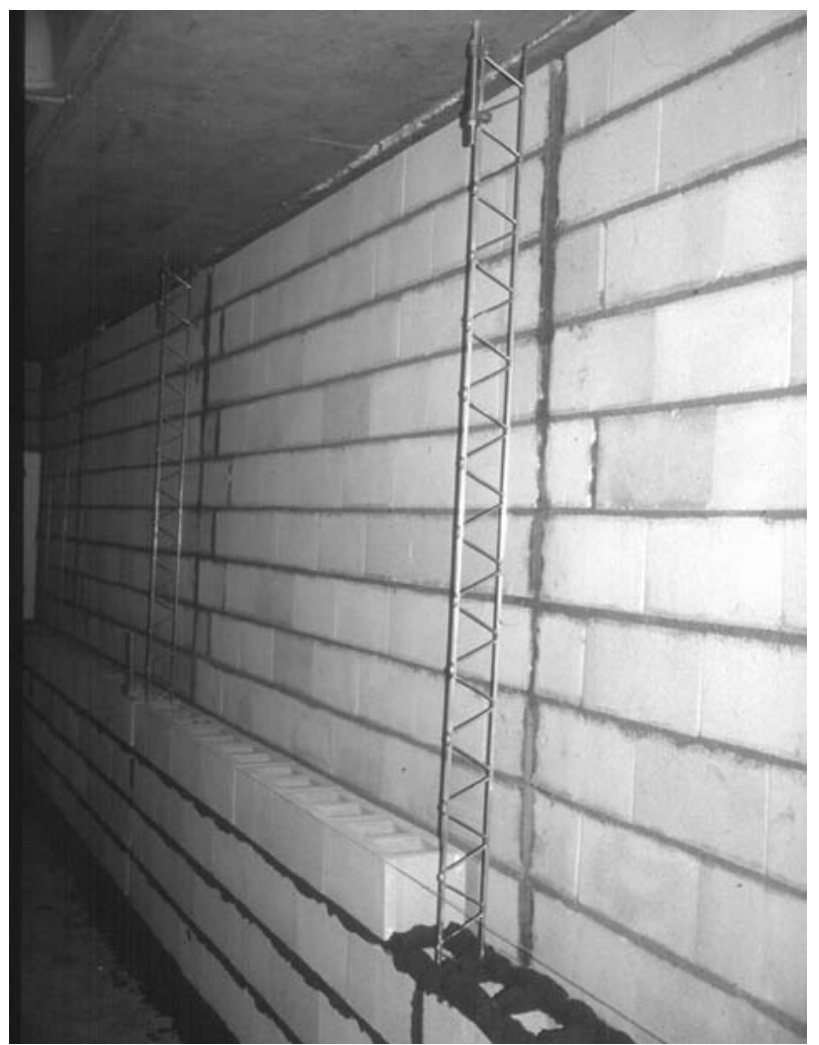

b)

Figura 6.- a) Muro con el Sistema de Albañilería Integral (SAI); b) Costillas AllWall distanciadas a 2,80 m entre sí, dispuestas en llaga vertical continua (en muro cortafuego doble del nuevo Aeropuerto de Madrid-Barajas).

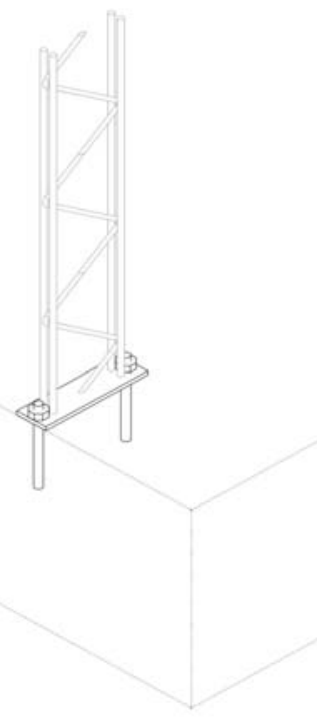

a)

a)

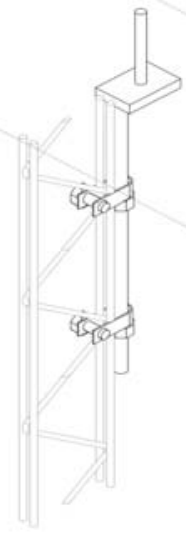

b)

Beta 2

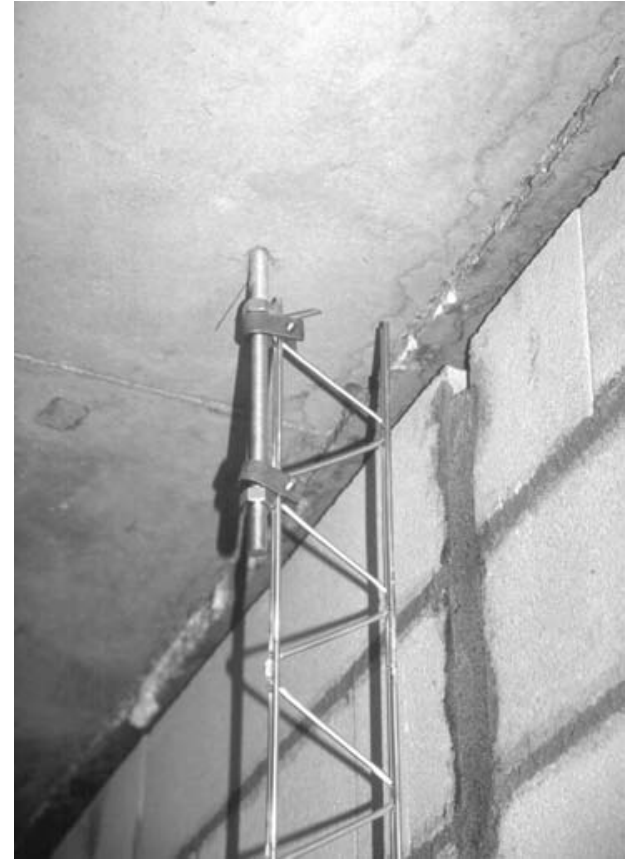

c)

Beta 1

Figura 7.- a) Fijación AllWall inferior Alfa; b) Fijación AllWall superior Beta 2; c) Fijación Telescópica de la Costilla AllWall al forjado superior Beta 1. 


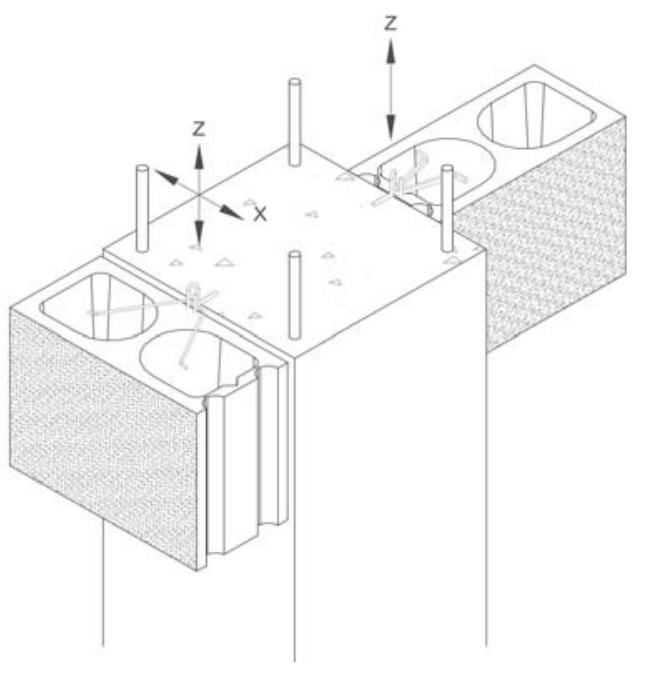

a)

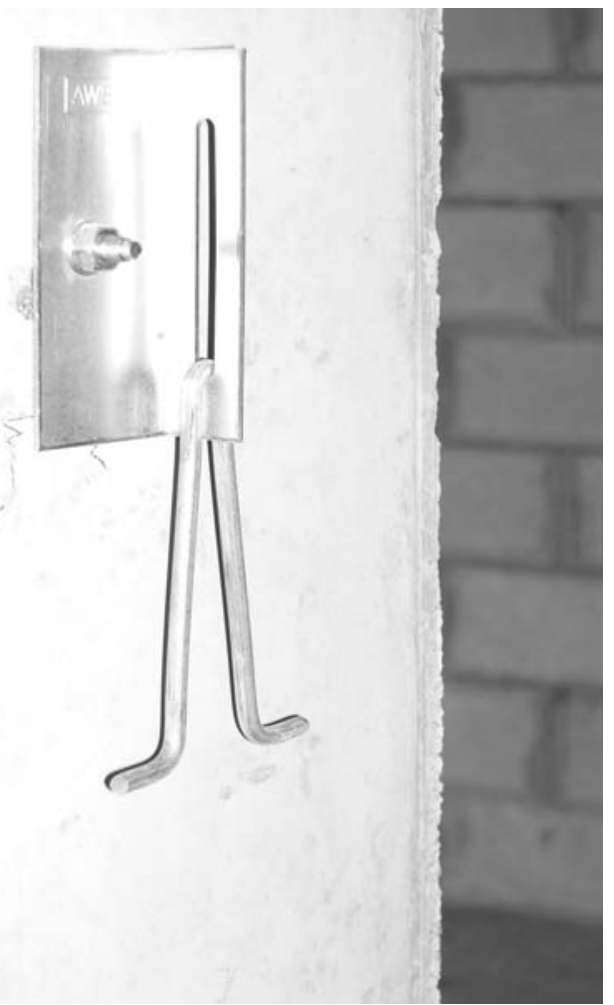

b)

Figura 8.- a) Gráfico de cerramiento pasante con 2 libertades de movimiento, vertical y horizontal AW-ANC.2 y de partición entestada con anclaje de una sola libertad de movimiento vertical $A W$-ANC.4.; b) Anclaje AW-ANC.4, con una sola libertad de movimiento vertical para partición entestada.

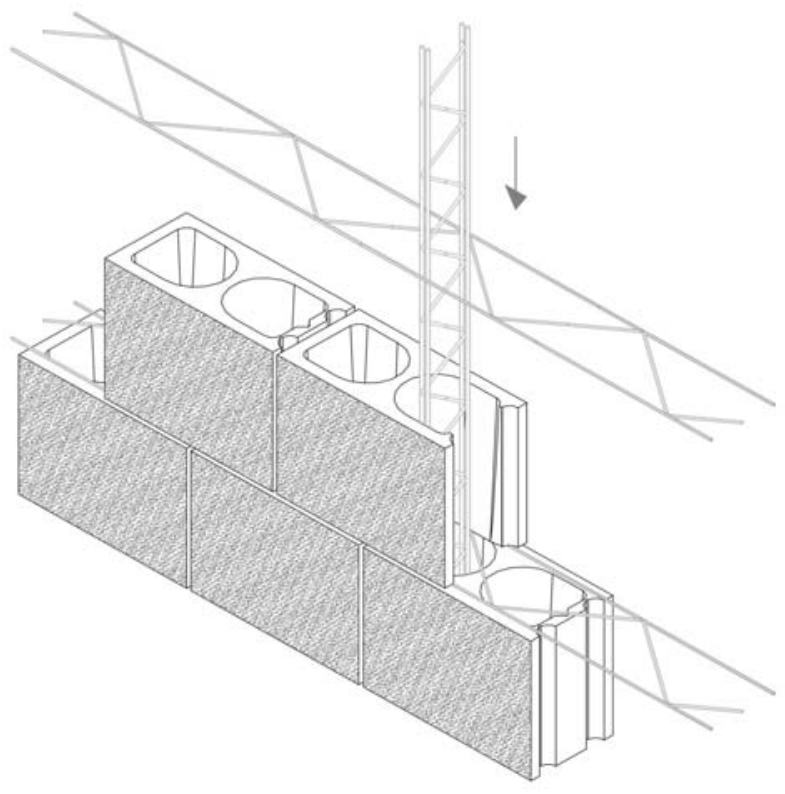

a)

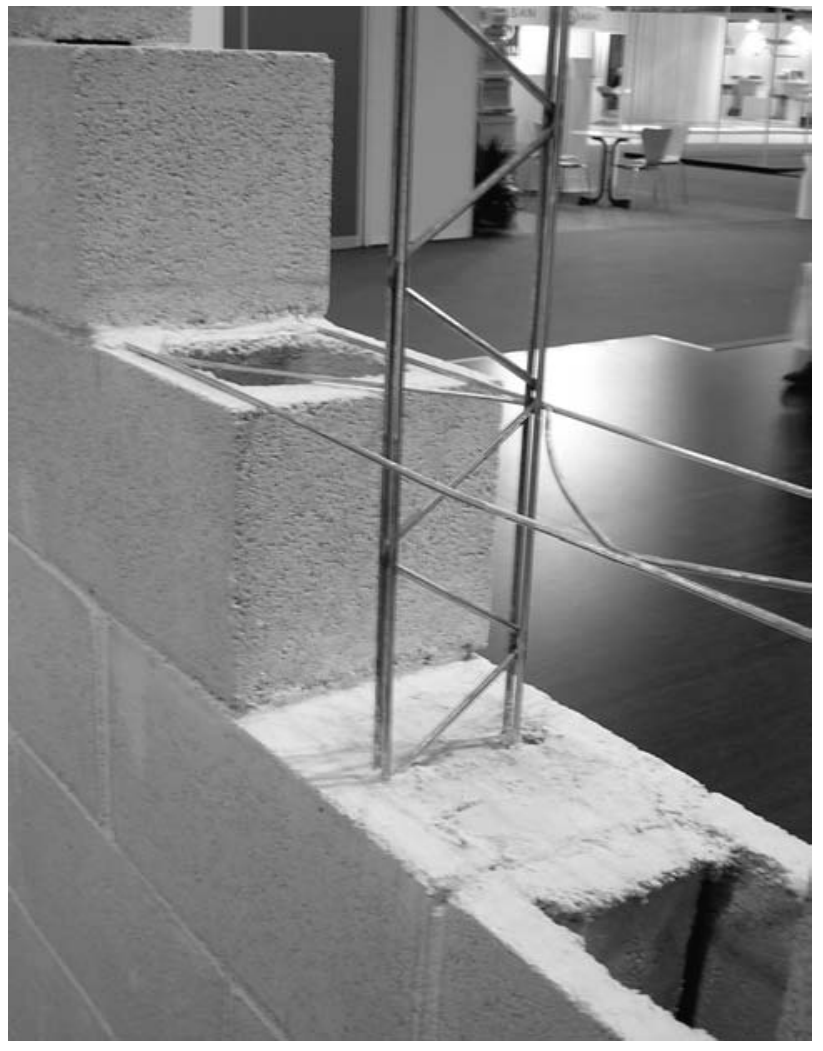

b)

Figura 9.- a) Gráfico de la Costilla ubicada dentro de la pieza BLOC+; b) Imagen de un caso de costilla embutida lateralmente dentro de la pieza del BLOC+. 


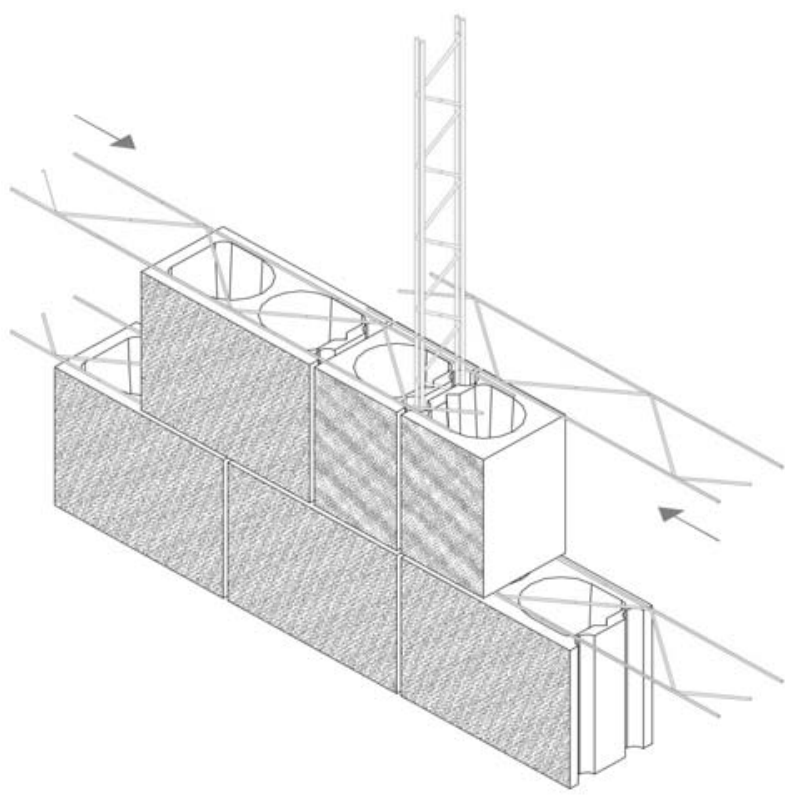

a)

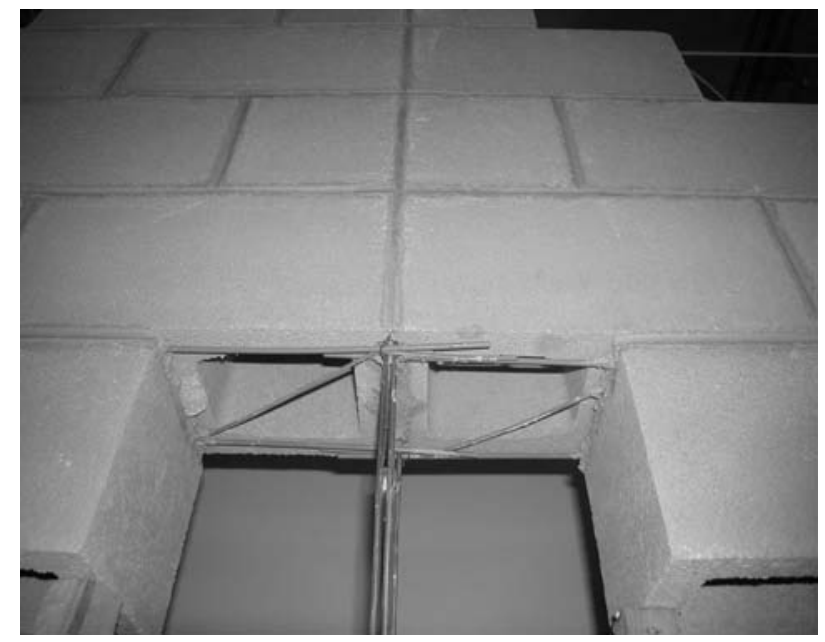

b)

Figura 10.- a) Gráfico de la Costilla ubicada en la llaga vertical continua; b) Vista de Costilla AllWall colocada en una llaga vertical continua en un muro de Construmat'03.


Figura 11.- a) Costillas verticales ya fijadas inferiormente, preparadas para construir un muro industrial; b) Costilla en llaga vertical continua, trabada con Murflor, en un muro de bloque para revestir de $6 \mathrm{~m}$ de alto. 
Lo que se señala en la Fig.11.b., de una fábrica para revocar, es la apariencia de una costilla vertical trabada en horizontal cada $60 \mathrm{~cm}$ de altura, en el centro de un paño de fábrica, lo que dista mucho de ser una junta vertical de movimiento entre dos paños de fábrica.

\section{APLICACIONES ARQUITECTÓNICAS ALLWALL BHH/BLOC+}

En todos los casos las ventajas del Sistema AllWall/BLOC+ están principalmente en que no se necesita hormigón armado, ya que las costillas tienen su sistema de fijación propio y son autorresistentes, soportando los esfuerzos perpendiculares a ellas, sin la necesidad de la contribución del hormigón que es lo habitual en los muros de bloque de hormigón rellenos de hormigón armado, con sus múltiples complicaciones técnicas.

Hay que resaltar que las diagonales de las cerchas de las costillas soportan el esfuerzo cortante en los apoyos, y que al ser las costillas galvanizadas, no se corroen al recubrirlas sólo con mortero.

Es muy habitual aplicar el Sistema AllWall/BLOC+ en muros de gran altura, interiores o exteriores, para edificios industriales o grandes superficies, o edificios singulares.

Se aplican costillas reforzadas, en grandes muros de 1 grueso hoja de BHH $(29 \mathrm{~cm})$, donde la ligereza de las costillas, inclusive reforzadas, permite una muy fácil manipulación, y donde no hace falta ningún relleno de hormigón armado (Fig.12.a,b).

En ocasiones, interesa hacer muros dobles de 2 hojas con un bloque más delgado $(14 \mathrm{~cm})$, donde las costillas abarcan el ancho de ambas hojas, atándolas entre sí junto con el armado horizontal, a caballo de ambas, también sólo con mortero, como en el caso del archivo de la Comunidad de Madrid en las Rozas (Fig.13.a,b).

Por el contrario, a veces en muros de gran altura, sobre todo si no pueden fijarse en la parte superior bajo la cubierta, se emplean muros con distinto grueso en la parte inferior que en la parte superior, combinando bloques de $19 \mathrm{~cm}$ en los $6 \mathrm{~m}$ de altura inferiores, con bloques de $14 \mathrm{~cm}$ en la parte superior, para economizar material.

En estos casos, se emplean las costillas apropiadas a cada grueso del paño de fábrica inferior $(150 \mathrm{~mm})$ y superior $(100 \mathrm{~mm})$. Y, al tratarse de un muro en ménsula, pueden disponerse las costillas más próximas en la parte inferior que en la superior, como se ha hecho en el Centro Comercial Eroski, en Vitoria (Fig.14).

El Sistema AllWall/BLOC+ ofrece las soluciones más idóneas en cualquier situación técnica y formal, como en
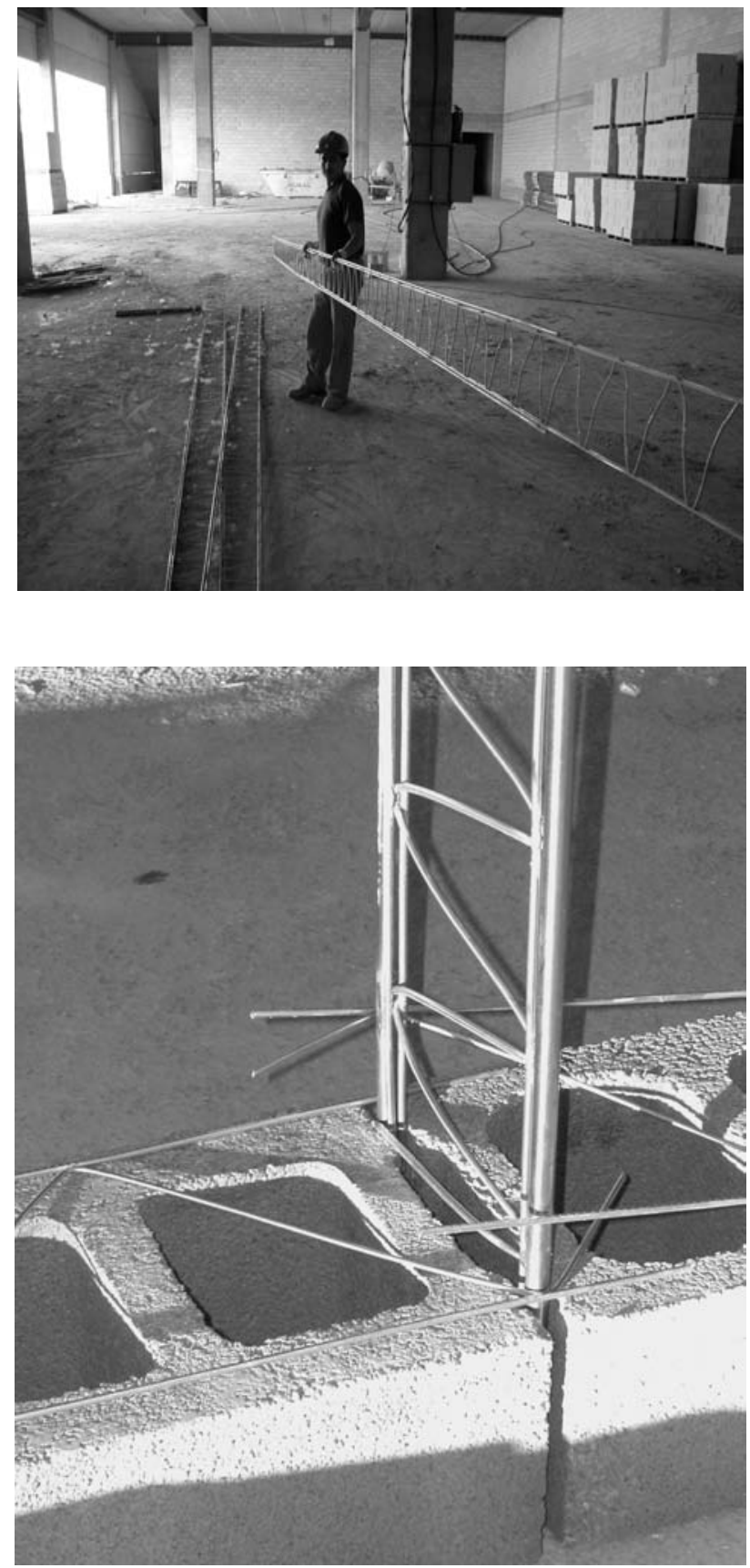

Figura 12.- a) Demostración de la ligereza de manipulación de una costilla reforzada de $8 \mathrm{~m}$ de altura; b) Replanteo de la costilla AllWall anterior de $200 \mathrm{~mm}$, con doble refuerzo, en un BHH de $29 \mathrm{~cm}$ de ancho.

el caso de los grandes muros y muros curvos del Hotel de las Bodegas de Marques de Riskal en El Ciego (Álava), diseñadas por el Arquitecto Ghery, y dirigidos por IDOM (Fig.15.a,b).

En este singular edificio y dadas las elevadas exigencias de acción de viento $\left(200 \mathrm{~kg} / \mathrm{m}^{2}\right)$, en muros de BHH de $19 \mathrm{~cm}$ y $7 \mathrm{~m}$ de alto, se ha necesitado emplear dobles costillas reforzadas y con refuerzo de cortante. También se han empleado en muros curvos de menor altura e igual presión, obligando a disponer las costillas cada 1,4 m (Fig.16.a,b). 

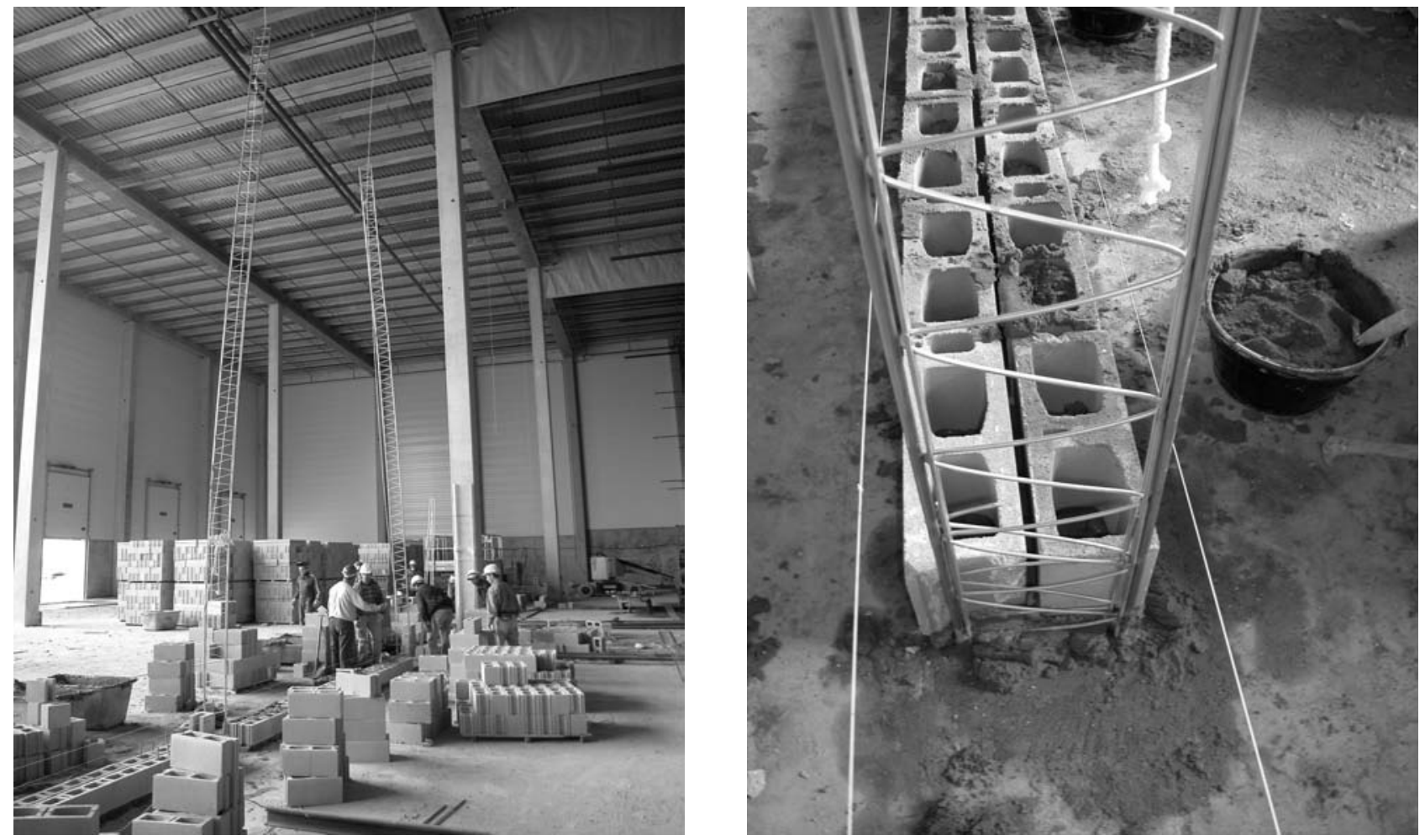

Fig.13.- a) Construcción con el SAI, de un muro doble interior de $15 \times 14 \mathrm{~m}$; b) Muro doble anterior con la costilla atando ambas hojas de BHH de $15 \mathrm{~cm}$ de grueso.

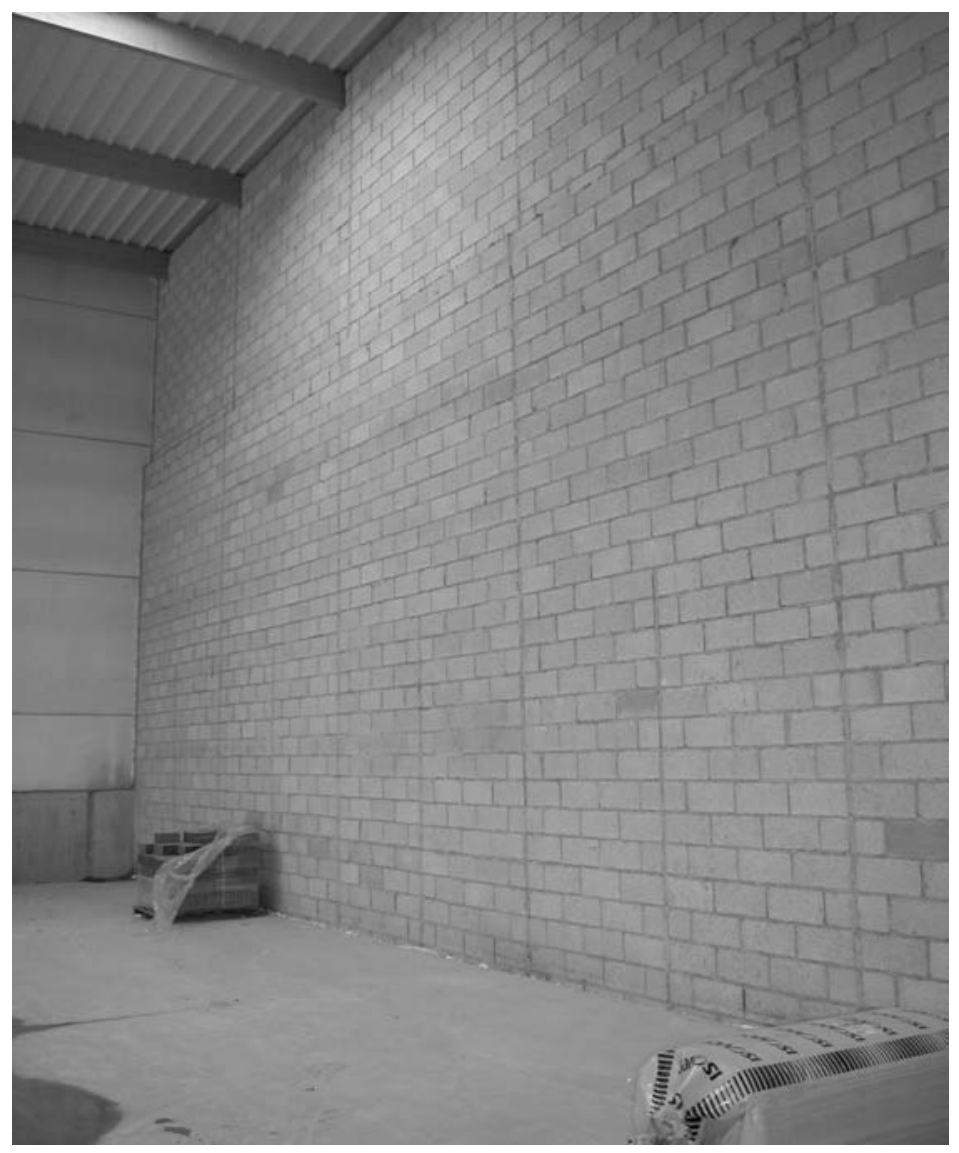

Figura 14.- Partición interior del Centro Comercial Eroski de Vitoria, empotrada abajo y libre arriba. Muro de $11 \mathrm{~m}$ de alto en 2 tramos, inferior con BHH de $29 \mathrm{~cm}$ y superior con BHH de $19 \mathrm{~cm}$, y con costillas de distinta altura para soportar el muro en ménsula. 

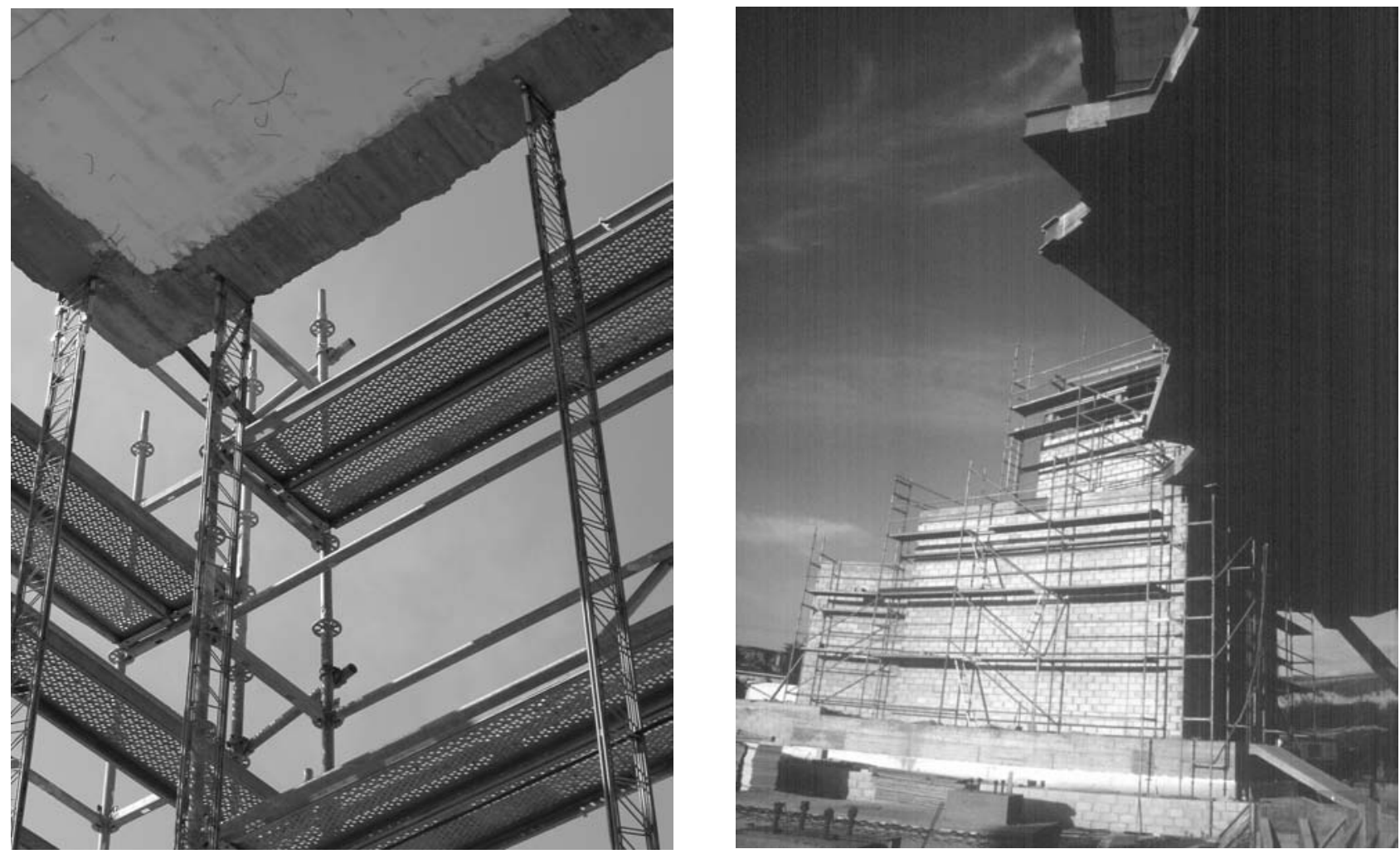

Figura 15.- a) Cerramiento del Hotel Marqués de Riskal en el proceso de ejecución de la fábrica con el SAI, empleando dobles costillas reforzadas de $7 \mathrm{~m}$ de alto; b) Muro terminado con costillas en llaga, a la espera del aislamiento y del chapado de piedra.
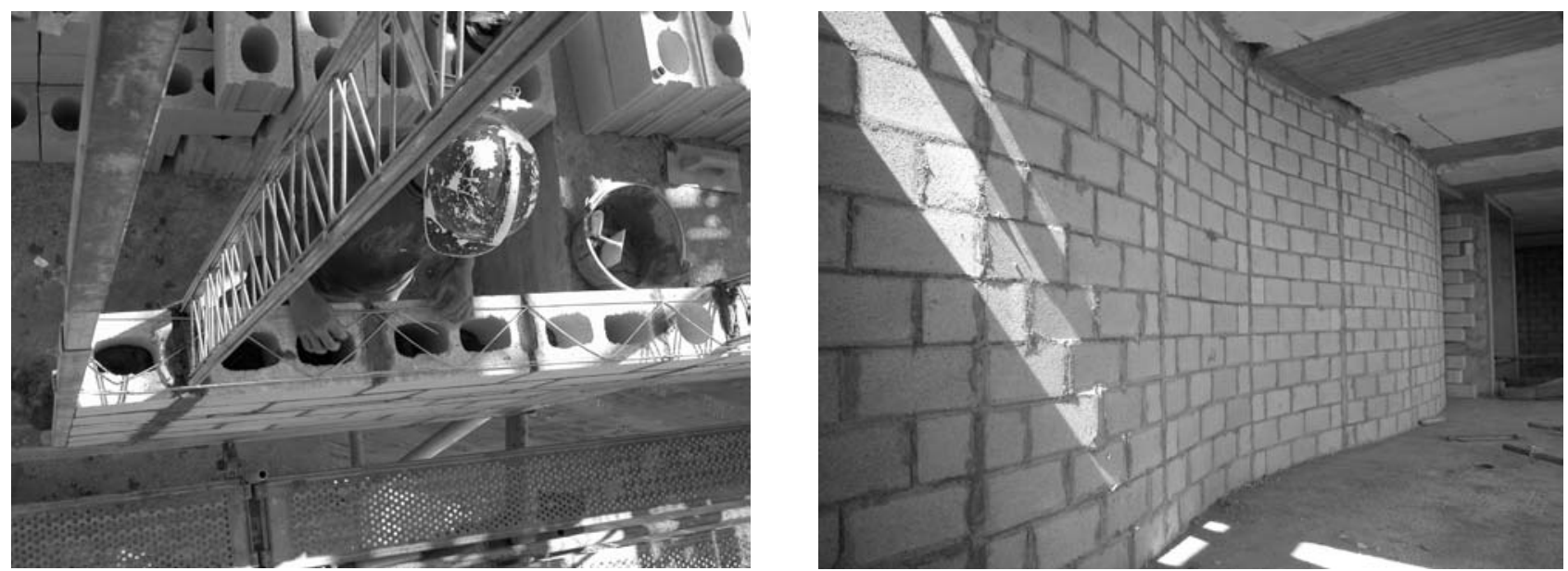

Figura 16.- a) Ejecución del cerramiento del Hotel Marqués de Riskal en un paño recto con dobles costillas reforzadas; $b$ ) Cerramiento de $3 m$ de altura con costillas en un paño curvo.

La imagen del edificio de Ghery, envolverá con chapados de piedra y de acero, los muros resistentes de bloque de hormigón, vistos anteriormente, con el Sistema AllWall (Fig.17). En España ya se han hecho muchos edificios con este sistema debido a sus múltiples ventajas, a las que añade la existencia de la asesoría Técnica AIA Arquitectura s.XXI, que vela por la adecuada explotación de la licencia de invención del Sistema AllWall. 


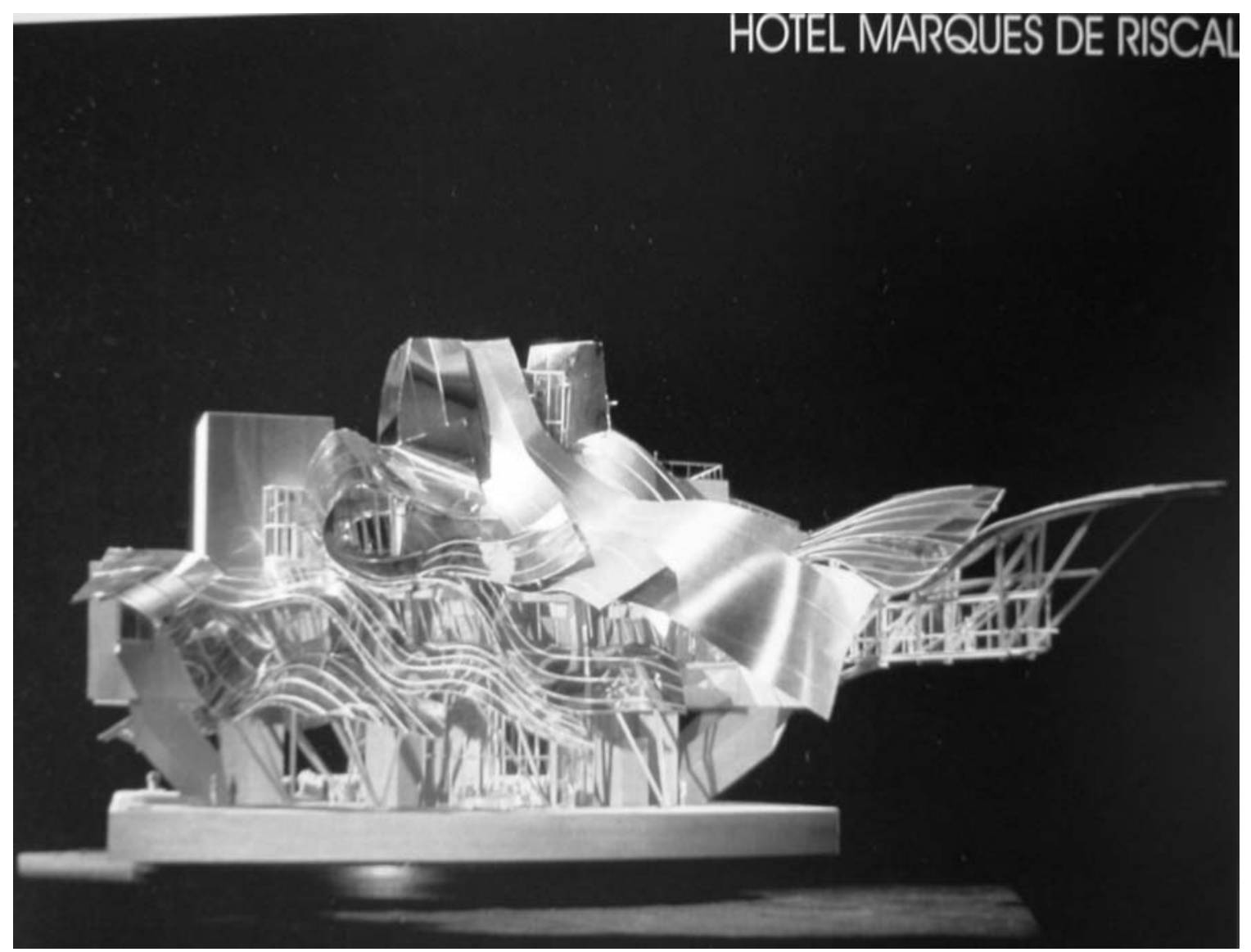

Figura 17.- Foto de la maqueta del Hotel Marqués de Riskal de F. O. Ghery, donde se emplea el SAI.

\section{BIBLIOGRAFÍA}

- Sobre la denominación de: "la fábrica armada". Adell, J.M. Actas II Congreso Hispanoamericano de Terminología de Edificación. Valladolid (1987).

- Arquitectura de investigación con fábrica armada ("Architecture and research with reinforced masonry"). Adell, J. M. Revista Informes de la Construcción. Vol. 44. n 421. Instituto de Ciencias de la Construcción Eduardo Torroja. (CSIC). Madrid, septiembre/octubre (1992).

- Razón y ser de la fábrica armada (Reason \& being of the reinforced masonry) Adell, J. M. Revista Informes de la Construcción. Vol. 44. $\mathrm{n}^{\circ}$ 421. Instituto de Ciencias de la Construcción Eduardo Torroja. (CSIC). Madrid, septiembre/octubre (1992).

- Manual Murfor: La Fábrica Armada. Adell, J. M; Lahuerta, J. A. (Cálculo). Bekaert Ibérica, S.A. Barcelona (1992) (reeditado en 2002).

La fábrica armada. Adell, J. M. Ed. Munilla-Lería. Madrid 2000. ISBN 84-89150-39-7.

- The integral masonry system and the contemporary façade. Adell, J. M. Paper of the $12^{\text {th }}$ International Brick/Block Masonry Conference. Madrid (2000).
- The universal masonry unit and rib reinforcement. Adell, J. M. Paper of the $12^{\text {th }}$ International Brick/Block Masonry Conference. Madrid (2000).

- Manuales AllWall: Sistema de Albañilería Integral: ("Allwall: Integral Masonry System”) Adell, J. M. Ed. AWS, AllWall Systems. Madrid entre los años 2001-2005.

- Bloque de hormigón hueco para todos los fabricantes de BHH (2002)

- Pieza Ferrater para Torho (2003)

- Brick Vallés para Calibloc (2003)

- Bloc+ (BlocPlus) para Normabloc (2005)

- Código de Buena Práctica para la ejecución de Fábricas con Bloques y Mampostería de Hormigón. Roces, C. Ed. Normabloc. Madrid (2004).

- Normabloc y BLOC+ en Construtec. Adell, J. M. Revista Editeco $\mathrm{n}^{\circ} 210$, diciembre (2004) (páginas 54 y 55).

- The Integral Masonry System. Adell, J. M. y Dávila, M D. $13^{\text {th }}$ IBMAC. Amsterdam, junio (2004) (páginas 519-528).

- Manual NORMABLOC: Asociación Nacional de Fabricantes de Bloques y Mampostería de hormigón. Adell, J. M. Ed. Normabloc. Madrid (2005) (en edición). 